\title{
Stockage géologique de déchets et droit du sous-sol : une fracture entre intérêt public et usage industriel ${ }^{\star}$
}

\author{
Thomas Schellenberger* \\ Droit public, Université de Haute-Alsace, Cerdacc, Colmar, France
}

\begin{abstract}
Résumé - Le développement du stockage géologique de déchets permet de s'interroger sur la façon dont le droit français appréhende le sous-sol et ce procédé qui génère des risques et des conflits d'usages à long terme. Le stockage géologique transfert les déchets dans un espace possédant des normes et des enjeux propres. Or, l'encadrement juridique du stockage souterrain de déchets tend à enfermer le sous-sol dans une fonction d'exutoire favorable à la production industrielle. Les règles de droit sur l'accès au sous-sol en sont une illustration: on peut dire qu'il existe une fracture, dans le droit du sous-sol, entre intérêt public et usage industriel. D'un côté, le sous-sol est approprié par l'État au nom de l'intérêt général. De l'autre, cet espace est mis à disposition des exploitants industriels au détriment d'un usage public du sous-sol envisagé plus largement.
\end{abstract}

Mots-clés : environnement / technologies / ressources naturelles / risques / changement climatique

\begin{abstract}
Legal framework for the geological disposal of waste: a divide between public interests and industrial use. As the geological storage of waste develops, the question arises about the French legal framework for the subsurface and these new activities which generate risks and conflicts of use in the long term. In order to better understand the legal issues involved in the geological disposal of waste, this technique needs to be studied as a process related to the currently existing industrial activities on the soil surface. Actually, the process of underground storage makes it possible to move the disadvantages of waste in space and time. Stored waste is not eliminated but moved to a new space which has its own standards. It should be emphasized that the legal framework for underground waste disposal tends to confine the subsoil to an evacuation function favorable to industrial production. The law on access to the geological space is an illustration of this. On the one hand, the geological space suitable for the storage of waste is appropriated by the State in the name of public interest. The geological storage spaces are therefore, like the mines, public goods. On the other hand, this same space is made available to industrial operators, which is restrictive as this is to the detriment of wider and diversified public uses. If the subsoil is appropriated by the State, in a certain way it also escapes the State for the benefit of industrial operators. It can therefore be said that there is a divide in the subsurface law between public interest and industrial use.
\end{abstract}

Keywords: environment / technologies / natural resources / risks / climate change

Le stockage géologique de déchets et de dioxyde de carbone $\left(\mathrm{CO}_{2}\right)$ en est à un point clé de son développement : alors que plusieurs procédés d'enfouissement sont en projet, certains sites profonds sont en phase de fermeture, et il s'agit déjà de pallier la défaillance de

\footnotetext{
‡ Cet article est une contribution au numéro coordonné par X. Arnauld de Sartre et S. Chailleux, intitulé «Pour une géologie politique $»$.

* Auteur correspondant :

thomas.schellenberger@uha.fr
}

certains autres ${ }^{1}$. Les opérations d'enfouissement géologique de déchets interrogent donc la société sur ses choix de développement passés, mais aussi sur ses directions à prendre aujourd'hui sur le long terme, à l'image des trois exemples suivants. La France a actuellement le projet d'enfouir à 500 mètres de profondeur les déchets

\footnotetext{
${ }^{1}$ Cet article a été rédigé à partir des résultats actualisés de la thèse soutenue par l'auteur (Schellenberger, 2014) pour laquelle il a obtenu le prix spécial de thèse 2015 de la Société française pour le droit de l'environnement.
} 
radioactifs les plus dangereux issus de son industrie nucléaire. C'est le projet Cigeo: $85000 \mathrm{~m}^{3}$ de ces déchets concentrant $99,8 \%$ de la radioactivité totale des déchets français, actifs pendant plusieurs centaines de milliers d'années, devraient normalement être stockés définitivement dans des galeries souterraines creusées dans le sous-sol de la Meuse, à Bure ${ }^{2}$. En outre, le développement massif du captage et stockage de $\mathrm{CO}_{2}$ est en plein questionnement en raison de certains échecs et de son potentiel économique incertain (Ineris, 2017). Un autre exemple frappant témoigne des enjeux du stockage souterrain de déchets: les galeries des anciennes mines de potasse d'Alsace (Haut-Rhin) ont accueilli 44000 tonnes de déchets industriels dangereux déposés à 500 mètres de profondeur par l'entreprise Stocamine. Or, après la fermeture précoce du site de Stocamine due à une fraude et à l'incendie d'une partie des déchets en 2002, l'État a décidé récemment de sceller définitivement ce site $^{3}$.

L'utilisation du sous-sol comme lieu d'évacuation des déchets permet de s'interroger sur la façon dont le droit appréhende cet espace convoité et ces activités nouvelles qui génèrent des risques et de potentiels conflits d'usages. Afin d'en saisir au mieux les enjeux juridiques, le stockage géologique de déchets doit être étudié comme un processus directement lié aux activités existant aujourd'hui en surface. On peut en effet partir du principe que le stockage géologique de déchets représente une décharge en ce sens qu'il permet de se «débarrasser d'une charge ${ }^{4}$. Cependant, les effets néfastes sur l'environnement des déchets stockés ne disparaissent pas pour autant. Le processus d'enfouissement souterrain permet de déplacer les déchets, et leurs inconvénients, dans l'espace et le temps. Il s'agit donc de comprendre dans quelles conditions est opéré ce transfert de la charge environnementale des déchets vers le soussol, en évaluant la capacité des cadres juridiques du stockage géologique à arbitrer entre les multiples intérêts en conflit dans les domaines de l'utilisation du sous-sol et de la gestion des déchets (exploitation industrielle, protection de l'environnement, gestion des risques et mise en valeur des ressources renouvelables).

Lorsque des déchets produits en surface sont transférés dans le sous-sol, on assiste à une transformation des cadres juridiques jusqu'alors en vigueur, les déchets étant intégrés à un nouvel espace possédant des normes propres. Une première illustration de cette

\footnotetext{
$\overline{2}$ https://www.andra.fr/cigeo.

${ }^{3}$ Communiqué de presse du préfet du Haut-Rhin, « Suites de la Commission de suivi de site (CSS) du 21 janvier 2019 relative au site de stockage illimité des déchets entreposés à Stocamine $»$.

${ }^{4}$ Entrée « décharge », in Dictionnaire de l'Académie française.
}

transformation peut être tirée des règles sur l'accès aux ressources du sous-sol. C'est cet aspect, focalisé sur l'appropriation juridique des ressources souterraines, qui sera approfondi dans le présent article. L'accès au soussol est principalement régi par le droit minier. Traditionnellement orienté vers la recherche d'une productivité industrielle stratégique pour la Nation, le droit minier est aujourd'hui appelé à régir le stockage de déchets et de $\mathrm{CO}_{2}$ qui sont pourtant au cœur d'enjeux environnementaux différents de ceux des mines puisqu'il s'agit d'évacuer dans le sous-sol des substances indésirables en surface. Comment sont organisés l'accès à cet espace et le partage des droits sur les ressources ? S'il peut y avoir un intérêt public à enfouir des déchets dans le sous-sol, ce dernier pourrait être considéré comme un espace public dont il conviendrait de déterminer les usages et finalités en fonction de l'intérêt général.

Or, on peut faire le constat que l'encadrement juridique de l'évacuation souterraine de déchets a tendance à enfermer le sous-sol dans une fonction d'exutoire favorable à la production industrielle. Lorsqu'on se place du point de vue de l'enfouissement de déchets, il existe en effet une tension, voire une fracture, dans le droit du sous-sol, entre intérêt public et usage industriel.

En effet, on peut considérer que le sous-sol est un bien public mis à disposition du secteur industriel. Il est en premier lieu un bien public. Fondée sur l'idée que le sous-sol contient des richesses à exploiter, l'appropriation des ressources souterraines par l'État supplante le droit de propriété privée du sous-sol tout en introduisant un objectif d'intérêt général dans l'accès au sous-sol. Il est en second lieu un bien mis à disposition du secteur industriel. Pour les besoins de la production de ressources, l'exploitant se voit en effet attribuer une place privilégiée dans l'accès au sous-sol. Mais si les instruments juridiques de l'État sont utilisés au profit d'une exploitation industrielle du sous-sol se pose la question de la garantie de l'intérêt général associé à la mise en valeur du sous-sol.

\section{L'appropriation publique de l'espace géologique apte au stockage de déchets}

Objet d'un droit de propriété, le sous-sol appartient en principe au propriétaire de la surface, mais les richesses économiques et stratégiques qu'il renferme donnent lieu à une appropriation par l'État. Il s'agit d'un axe central $\mathrm{du}$ droit du sous-sol qui implique un accès public prioritaire aux ressources, restreignant ainsi la propriété privée au nom de l'intérêt général attaché à la mise en valeur des ressources souterraines. 


\section{Le principe du prolongement de la propriété de la surface en sous-sol}

Il existe en droit civil français une présomption de rattachement du tréfonds à la propriété de la surface prévue par l'article 552 du code civil selon lequel « la propriété du sol emporte la propriété du dessus et du dessous [...]». Par conséquent, «tout ce qui est dans le sous-sol est présumé être rattaché à la propriété de la surface [...] (Billet, 1994), sans limite de profondeur. Deux conséquences en matière d'accès au sous-sol découlent de cette règle de propriété par accession selon laquelle le sous-sol, c'est-à-dire l'accessoire, est automatiquement rattaché à la propriété d'un bien principal, le fonds en surface.

D'une part, c'est en vertu de ce droit de propriété que la législation minière accorde au propriétaire du sol le droit d'effectuer librement des recherches dans son tréfonds. Cette liberté d'accès au sous-sol est prévue par l'article L. 121-1 du code minier qui dispose que les travaux de recherches de mines peuvent être entrepris «par le propriétaire ou avec son consentement». On verra plus loin que la découverte de gisements de matières exploitables entraîne la perte de la propriété du tréfonds au profit de l'État. Mais la présomption de propriété dont bénéficie le propriétaire de la surface ne tombe qu'au moment de l'institution d'une concession de mine. Autrement dit, et c'est un principe très important, tant qu'un gisement exploitable n'a pas été découvert, le propriétaire de la surface est présumé propriétaire du sous-sol.

D'autre part, le principe de la propriété du sous-sol par accession s'applique également à l'exploitation des carrières. La réglementation des carrières reconnaît en effet un droit de propriété sur les ressources souterraines dont le propriétaire de la surface est titulaire. Selon l'article L. 332-1 du code minier, «les carrières sont laissées à la disposition du propriétaire du sol». Aussi le principe de la libre disposition des ressources souterraines au profit du propriétaire de la surface oblige-t-il le pétitionnaire à acquérir la maîtrise foncière du site convoité ou à obtenir le consentement du propriétaire de la surface (Gillig, 2009) ${ }^{5}$.

Le principe de l'attribution de la propriété du sous-sol au propriétaire de la surface est prédominant par exemple aux États-Unis. Bien que les droits de propriété sur le sous-sol en matière de stockage souterrain de déchets varient en fonction des États fédérés (Campbell et al., 2008), des orientations juridiques destinées à harmoniser le droit américain de la propriété des sites souterrains de stockage de $\mathrm{CO}_{2}$ ont été rédigées sur la base des droits

\footnotetext{
${ }^{5}$ Cour administrative d'appel de Nancy, 04/12/2008, $\mathrm{n}^{\circ} 06$ NC01641, M. X et Assoc. pour la sauvegarde du sol et du sous-sol.
}

existant dans la plupart des États ${ }^{6}$. Deux principes en ressortent. D'une part, le sous-sol doit appartenir au propriétaire de la surface qui peut le céder, le louer ou bien céder les droits liés à son utilisation comme site de stockage à un tiers. D'autre part, ladite commission préconise qu'en cas de refus de la part du propriétaire de la surface d'accorder au pétitionnaire d'un projet de stockage de $\mathrm{CO}_{2}$ le droit de stockage, le gouvernement puisse exproprier la partie du tréfonds nécessaire au stockage au bénéfice du pétitionnaire (Grave, 2010). L'accès aux couches géologiques de stockage par accession à partir du droit de propriété de la surface est également la méthode qui a été utilisée par l'Andra pour mettre en place le projet Cigeo. Celle-ci a en pratique systématiquement procédé à l'achat amiable des fonds au sein desquels les galeriers de stockage doivent être aménagées. En cas de refus de vente par le propriétaire, le code de l'environnement, et non le code minier, prévoit que les terrains nécessaires à l'implantation du stockage pourront être expropriés (art. L. 542-8). Le cas de Cigeo est une exception par rapport à l'ensemble des sites français de stockage de déchets ou de $\mathrm{CO}_{2}$. Cigeo est fondé sur un régime juridique ad hoc lié au caractère exceptionnel de ce projet qui, sur le plan du droit de propriété, n'est pas comparable aux autres sites de stockage de déchets.

Les ressources souterraines peuvent donc en principe être rattachées à la propriété de la surface. Toutefois, ce principe tiré du code civil ne s'applique, en droit français, qu'au stade des recherches de mines ou aux carrières. Si le droit américain du stockage souterrain de fluides se fonde sur une logique comparable, il convient de souligner qu'à l'exception du projet Cigeo mentionnée ci-dessus c'est un tout autre système qui est applicable en droit français pour encadrer les stockages géologiques de déchets.

\section{L'appropriation des richesses souterraines par l'État}

Le code minier distingue deux grands régimes relatifs à l'exploitation du sous-sol: celui des mines et celui des carrières. Cette distinction repose sur la valeur marchande ou stratégique des matières considérées. D'un côté, les ressources minières sont vues comme des « richesses naturelles » (Laubadère, 1977) vitales pour la Nation (Gauché, 2003) dont la propriété est attribuée à l'État (Terré et Simler, 2006). C'est ce qui explique pourquoi les gisements carriers, réputés d'une importance économique moindre, sont rattachés à la propriété

\footnotetext{
6 IOGCC, 2007, Storage of carbon dioxide in geologic structures: a legal and regulatory guide for states and provinces - Documents.OK.Gov - Oklahoma Digital Prairie: Documents, Images and Information.
} 
immobilière du sol. On peut ajouter que cette structure reposant sur la notion de richesse nationale à valoriser est constante au vu des propositions de réforme du code minier qui ont pu être faites ${ }^{7}$.

Comme le postule William Dross (2010) : «On aurait pu s'attendre à ce que la législation minière se contente de réglementer l'extraction des minerais [...] afin que l'intérêt général soit préservé. [...] La réalité est tout autre, car dès 1810 le système mis en œuvre procède à une véritable dissociation de l'immeuble en tant qu'objet du droit de propriété. La mine est séparée du sol, de la superficie et du reste du tréfonds pour être appropriée directement par l'État.»

Quant à Philippe Billet (1994), il précise que «les gisements miniers sont distraits de la propriété du sol et, quel que soit leur mode d'exploitation, ils demeurent la propriété de l'État». Cette solution paraît confirmée par la jurisprudence relative à l'expropriation du tréfonds : la notion de « richesse nationale » permet en effet de fonder un régime de propriété étatique où les ressources souterraines sont exploitées via des concessions délivrées par l'État, comme en a depuis longtemps pris acte la littérature technique (Cuvillier, 1902).

Il convient toutefois de préciser que l'appropriation étatique des mines n'est pas prévue expressément par le code minier, elle résulte d'analyses théoriques (Levy et Castaldo, 2002) et de décisions de justice. En effet, Philippe Billet (1994) explique que le droit de propriété souterrain «échappe dans tous ses éléments au propriétaire du sol, faisant ainsi tomber la présomption de l'article 552 [du code civil] au profit de l'État». Le gisement minier n'appartient donc ni au propriétaire de la surface ni à l'exploitant. Trois indices délivrés par le code minier $^{8}$ permettent généralement d'étayer cette thèse. D'abord, la concession de mine n'est que provisoire. Puisque ce titre minier est attribué pour une durée déterminée par l'État à l'exploitant, ce dernier ne peut pas être considéré comme étant propriétaire du gisement souterrain. Ensuite, l'État participe aux bénéfices générés par l'exploitation de la mine via une redevance. Enfin, le gisement minier fait gratuitement retour à l'État à l'échéance de la concession (Personnaz et al., 2004b). La jurisprudence administrative se fonde également sur ces trois indices. En effet, le Conseil d'État dans un arrêt du 19 avril 2011 a confirmé que la délivrance d'une concession d'un gîte géothermique

\footnotetext{
${ }^{7}$ Proposition de réforme du code minier émise par T. Tuot, conseiller d'État désigné par le Premier ministre, juillet 2013 : «Article L. 111-1 : Le sous-sol relevant de la compétence des pouvoirs publics au titre et dans les limites du présent code est une richesse qu'il leur appartient dans l'exercice des pouvoirs relevant de cette compétence de valoriser et de préserver (...)». ${ }^{8}$ Articles L. $132-8$ et L. 132-13 du code minier.
}

n'était en rien une atteinte à la propriété privée, car, justement, la ressource minière appartient, du seul fait de sa découverte, à l'État ${ }^{9}$. Il a été rappelé à cette occasion que «l'institution d'une concession, même au profit du propriétaire de la surface, [créait] un droit immobilier distinct de la propriété de la surface » et que « le gisement [faisait] retour gratuitement à l'État» en fin d'exploitation, ce qui exclut le propriétaire de la surface de toute appropriation de la ressource géothermique.

Il est alors remarquable de constater que l'espace géologique apte au stockage de déchets a été assimilé juridiquement à des mines, soit expressément par la loi, soit de facto par la pratique, ce qui entraîne son appropriation par l'État (Schellenberger, 2014). Le régime du stockage géologique de $\mathrm{CO}_{2}$ en est un exemple. En principe, les gisements de substances concessibles décrits à l'article L. 111-1 du code minier, c'est-à-dire les mines, renvoient principalement aux ressources minérales et aux hydrocarbures. Cependant, sont également soumises au régime des mines les «cavités et formations [...] naturelles ou artificielles [...] présentant les qualités requises pour constituer des réservoirs [...] en vue du stockage de gaz naturel, d'hydrocarbures liquides, liquéfiés ou gazeux ou de produits chimiques à destination industrielle ». Bien que le stockage de déchets ou de $\mathrm{CO}_{2}$ ne soit pas mentionné dans ce texte, c'est sur ce fondement que le premier site pilote de stockage de $\mathrm{CO}_{2}$ a été autorisé par l'État en 2009 (Schellenberger, 2014). Mais depuis l'ordonnance 2010-1232 du 21 octobre 2010, les formations géologiques aptes au stockage de $\mathrm{CO}_{2}$ sont expressément soumises au régime des mines ${ }^{10}$. Comme dans le cas du stockage stratégique de matières premières susmentionné, ce sont donc les propriétés physiques de l'espace de stockage qui sont concédées et non une matière à extraire. En pratique, pour le stockage de $\mathrm{CO}_{2}$, c'est la roche poreuse composant les aquifères sédimentaires qui deviendra concessible. Puisqu'aucune matière n'est désignée en particulier, toute formation géologique capable de constituer un réservoir en vue de stockage de $\mathrm{CO}_{2}$ devient donc une matière concessible au titre du code minier, ce qui élargit les droits potentiels d'accès au sous-sol pour y stocker des résidus de production industrielle.

Les espaces géologiques de stockage sont donc des biens publics, tout comme les mines, en raison de leur intérêt économique pour la collectivité nationale. De ce fait, ni l'achat amiable du tréfonds ni son expropriation ne seront nécessaires pour accéder aux sites souterrains. Le transfert vertical des déchets entraîne une réindustrialisation du sous-sol à partir de la surface. Il s'agit d'une forme d'exploitation minière inversée où le

\footnotetext{
${ }^{9}$ Conseil d'État, 19 avril $2011, \mathrm{n}^{\circ} 332140$.

${ }^{10}$ L. 229-37 et L. 229-36 du code de l'environnement.
} 
sous-sol redevient une ressource industrielle, mais en tant qu'exutoire pour les activités en surface.

Si le sous-sol échappe au propriétaire de la surface pour être approprié par l'État, il échappe aussi dans une certaine mesure à l'Etat au profit des exploitants du soussol.

\section{La mise à disposition du sous-sol au profit des exploitants miniers}

L'État s'approprie le sous-sol pour le mettre à disposition des exploitants industriels et souvent privés, au profit de l'économie nationale. Mais dès lors que la puissance publique est sollicitée, la préservation de l'intérêt général doit être recherchée dans l'utilisation du sous-sol. Parallèlement, la protection des intérêts industriels est également assurée pour des raisons d'attractivité économique. Pommier (1939) parlait, au sujet des titres miniers, d' "une sorte de permissionobligation », car l'État souhaite à la fois favoriser la mise en valeur des ressources souterraines tout en optimisant les effets sur l'économie nationale (Personnaz et al., 2004a). Or, aujourd'hui, il existe un risque de déséquilibre entre les intérêts publics et privés fragilisant la protection de l'intérêt général.

En effet, l'accès au sous-sol est réglé par la délivrance de titres miniers instituant des droits sur les ressources souterraines. La recherche de l'intérêt général justifie le rôle central de l'État si bien que le sous-sol n'est pas livré «clé en main» aux industriels. Cependant, la nature et l'étendue des droits créés par les titres miniers sont susceptibles d'aller contre les intérêts publics en réduisant la place et les pouvoirs de l'État. D'un côté, les titres miniers entraînent des droits importants au profit de leurs titulaires. De l'autre, les effets d'entraînement nés des garanties juridiques accordées aux exploitants en matière de droits d'exploitation vont réduire la marge de manœuvre de l'État.

\section{L'institution de droits importants pour les exploitants}

Pouvant être localisées jusqu'à plusieurs milliers de mètres de profondeur, les couches géologiques favorables au stockage doivent, pour être découvertes, faire l'objet d'un travail de recherche. La délivrance d'un titre minier d'exploration précède donc l'institution d'une concession d'exploitation. Or, la loi sécurise solidement l'installation des industriels en sous-sol durant les recherches et l'exploitation. D'un côté, l'explorateur minier se voit attribuer un droit de recherche privilégié et, de l'autre, d'importantes prérogatives de puissance publique sont prévues pour faciliter l'accès aux ressources du sous-sol.
Un droit de recherche privilégié est en effet institué au profit de l'opérateur minier qui entreprend des recherches. Comme présenté plus haut, le droit d'effectuer des recherches souterraines appartient en principe au propriétaire de la surface, seule l'institution d'une concession met un terme à cette présomption de propriété. Cependant, avec le développement du stockage géologique de déchets et en particulier du $\mathrm{CO}_{2}$, un instrument juridique s'est imposé dans le code minier : le permis exclusif de recherche. Or, l'institution d'un permis exclusif de recherche permettra à l'explorateur d'évincer le propriétaire de la surface pour accéder directement aux ressources souterraines avant l'obtention d'une concession. Ce privilège est issu de la réglementation pétrolière ${ }^{11}$. Instrument dérogatoire du droit commun minier (Sillard, 1955), le permis exclusif de recherche a pour but d'accélérer l'exploration des hydrocarbures liquides et gazeux, ressources particulièrement convoitées et dont la recherche est considérée comme chère et aléatoire (Devaux-Charbonnel, 1987). Aujourd'hui, le code de l'environnement prévoit que les recherches de formations géologiques aptes au stockage de $\mathrm{CO}_{2}$ ne peuvent être entreprises qu'en vertu d'un permis exclusif de recherche ${ }^{12}$. De ce fait, deux privilèges principaux seront conférés au titulaire de ce titre de recherche. D'une part, ce titre «confère à son titulaire l'exclusivité du droit d'effectuer tous travaux de recherches dans le périmètre qu'il définit ${ }^{13}{ }^{13}$. De ce fait, le droit initial de recherche du propriétaire de la surface disparaît. L'utilisation du permis exclusif de recherche en matière de stockage de $\mathrm{CO}_{2}$ écarte ainsi totalement le propriétaire de la surface et renforce la place de l'explorateur. D'autre part, l'explorateur minier a le droit de «disposer librement des produits extraits à l'occasion des recherches et des essais ${ }^{14}$. La commercialisation des produits extraits du sous-sol à l'occasion des recherches est classiquement interdite par le code minier pour éviter les cas d'exploitation déguisée, qui entreraient en conflit avec le droit de propriété et nuiraient au contrôle étatique de l'activité minière. Toutefois, la réglementation pétrolière aujourd'hui applicable au stockage de $\mathrm{CO}_{2}$ déroge à cette interdiction. C'est avec la nécessité économique et stratégique d'encourager la recherche d'hydrocarbures que le code minier a assoupli cette interdiction, permettant à l'explorateur de disposer librement des hydrocarbures

\footnotetext{
${ }^{11} \mathrm{Le}$ régime $\mathrm{du}$ permis exclusif de recherche figure aujourd'hui aux articles 7 à 19 du code minier et aux articles 17 s. et $43 \mathrm{~s}$. du décret $\mathrm{n}^{\circ} 2006-648$ du 2 juin 2006 relatif aux titres miniers et aux titres de stockage souterrain, $J O R F \mathrm{n}^{\circ} 128$ du 3 juin 2006, p. 8414 .

${ }^{12}$ C. env., art. L. 229-30.

${ }^{13}$ C. min., art. L. 122-1, par renvoi de l'article L. 229-30 du code de l'environnement.

${ }^{14}$ Ibid.
} 
extraits lors de ses recherches (Devaux-Charbonnel, 1987).

L'opérateur minier pourra aussi bénéficier de prérogatives de puissance publique importantes lui permettant de mettre avantageusement en valeur le sous-sol. Ces privilèges apparaissent encore plus forts que ceux prévus par le régime des travaux publics applicable au stockage de déchets radioactifs ${ }^{15}$. Selon le code minier, l'opérateur minier pourra bénéficier, sur autorisation administrative, du droit d'occuper la surface et le sous-sol ainsi que celui d'y exercer des servitudes. Prévus aux articles L. 153-1 et suivants du code minier, les privilèges de l'opérateur minier s'appliquent aussi bien à la période d'exploitation qu'à la période de recherche. Selon l'article L. 229-30 du code de l'environnement, l'exploitant, mais aussi l'explorateur d'un site de stockage de $\mathrm{CO}_{2}$ bénéficient des mêmes prérogatives que l'exploitant minier. Il en va de même pour l'exploitant ou l'explorateur d'un site de stockage de déchets ultimes en vertu de l'article L.541-20 du même code. Matériellement, l'opérateur pourra obtenir le droit d'occuper en surface tous «les terrains nécessaires à l'exploitation de sa mine» et à l'implantation des dépendances minières ${ }^{16}$. De même, l'opérateur minier pourra être autorisé à dresser et enterrer des câbles et des canalisations, dégager le sol de tous arbres, arbustes ou autres obstacles ${ }^{77}$. Dès lors qu'il est titulaire d'un titre de recherche ou d'exploitation, l'opérateur minier bénéficie donc en sous-sol de droits plus forts que ceux pouvant être attribués à un industriel en surface.

\section{Les effets d'entraînement favorables aux futurs exploitants}

Dans le code minier, il existe des effets d'entraînement qui permettent la consolidation difficilement réversible des droits des exploitants, afin de faciliter leur accès aux ressources. Les garanties attribuées aux opérateurs miniers réduisent alors sensiblement le pouvoir d'appréciation de l'État dans l'octroi des droits d'accès au sous-sol. Le rôle de l'État dans la régulation de l'accès au sous-sol s'en retrouve amoindri. Cette remarque vaut d'une part pour la reconduction automatique de la validité du permis exclusif de recherche et d'autre part pour le droit quasi automatique à exploiter la formation géologique découverte.

\footnotetext{
${ }^{15}$ Les recherches et 1 'exploitation du site de stockage géologique des déchets radioactifs s'effectuent selon un régime dérogatoire et indépendant du code minier prévu par la loi du 29 décembre 1892 sur les dommages causés à la propriété privée par l'exécution des travaux publics (Schellenberger, 2014).

${ }^{16}$ C. min., art. L. $153-1$ et s.

${ }^{17}$ C. min., art. L. 153-8.
}

Le permis exclusif de recherche offre en effet à l'opérateur minier « une situation mieux définie et plus stable qu'un[e] simple (...) autorisation administrative » (Personnaz et al., 2004a). On s'aperçoit en effet qu'une fois institué, ce titre minier peut être prolongé plusieurs fois de plein droit ${ }^{18}$. Or, lorsque le renouvellement d'un titre est acquis de plein droit, le pouvoir de l'administration recule. On peut mentionner en particulier la dernière prolongation automatique d'une durée de quinze mois qui couvre la période séparant l'expiration normale du titre de recherche et la décision administrative portant sur la demande de concession minière formulée par l'explorateur ${ }^{19}$. Ce qui est frappant ici, c'est que le silence gardé par l'administration sur la demande de concession équivaut à un accord tacite de prorogation temporaire du titre de recherche, marquant ainsi la position de force de l'explorateur et le recul des pouvoirs de l'administration en la matière.

Ce qu'il faut souligner, c'est que les effets juridiques $\mathrm{du}$ permis exclusif de recherche sont d'autant plus importants que ce titre garantit le droit d'exploiter la formation géologique découverte. En effet, le droit quasi automatique d'exploiter le gisement découvert, qui s'est progressivement généralisé, relativise la place de l'État dans l'accès aux ressources souterraines. Fondé sur l'impératif économique d'accéder aux ressources pétrolières, ce principe est aujourd'hui transposé notamment au stockage géologique de $\mathrm{CO}_{2}$ et induit des effets importants sur les pouvoirs de l'autorité publique. Historiquement, l'apparition du permis exclusif de recherche d'hydrocarbures en 1922 a fait naître le droit pour l'explorateur d'exploiter le gisement découvert $\left(\right.$ Pommier, 1939; Sillard, 1955) ${ }^{20}$. Le droit à obtenir une concession minière procédait de l'idée qu'un avantage devait être accordé au permissionnaire en contrepartie des dépenses consenties (Personnaz et al., 2004b). L'article L. 132-6 du code minier prévoit donc un droit à l'octroi d'une concession minière au bénéfice de l'explorateur, constituant encore aujourd'hui une incitation économique décisive pour le secteur minier: "Les sommes engagées durant la phase de recherches ne seront en effet amorties que si l'opérateur a la certitude qu'il pourra exploiter le gisement plusieurs années après » (Gautier, 2013).

L'article L. 132-6 du code minier est le signe d'un recul de l'État, car il diminue le pouvoir d'appréciation de ce dernier lors de l'attribution des concessions

\footnotetext{
${ }^{18}$ Fixée initialement à cinq ans par l'article L. 122-3 du code minier, la durée de validité du permis de recherche peut être prolongée de plein droit pour une période de deux fois trois ans en vertu de l'article L. 142-1 du même Code.

${ }^{19}$ C. min., art. L. $142-1$ et L. $142-4$.

${ }^{20} \mathrm{Ce}$ droit est issu de la loi du 16 décembre 1922 ainsi que du décret-loi du 17 juin.
} 
minières. On observe alors que le rôle des concessions de mine, qui sont en principe centrales dans le droit minier, recule au profit de droits "acquis» dès la phase de recherche. En effet, historiquement, la concession constituait «le point culminant de l'intervention administrative» (Vidal, 1949). Cependant, l'article L. 132-6 du code minier réduit le pouvoir d'appréciation de l'administration lors de la délivrance des concessions de stockage. Dès lors qu'il est conditionné notamment à la démonstration par le demandeur du caractère exploitable de la ressource découverte, le droit d'obtenir une concession d'exploitation n'est pas juridiquement une garantie et n'est donc pas, a proprement dit, automatique. Toutefois, l'obtention quasi automatique d'une concession sur le sous-sol n'en demeure pas moins un «droit très important » (Laubadère, 1977), facteur de limitation des pouvoirs de l'administration. En matière de stockage de $\mathrm{CO}_{2}$ par exemple, le ministre compétent est en effet tenu de délivrer la concession de stockage géologique demandée si les conditions environnementales et de sécurité mentionnées aux articles L. 229-37 du code de l'environnement et L.132-6 du code minier sont remplies.

Finalement, le droit prévu à l'article L. 132-6 du code minier confère en réalité au permis exclusif de recherche une position prédominante. En accordant un permis exclusif de recherche, l'État "s'engage déjà à accorder une concession » (Pommier, 1939) et donc la privation de propriété du propriétaire de la surface se fait, en quelque sorte, par anticipation. Paradoxalement, c'est donc l'octroi du permis exclusif de recherche qui devient central, alors que celui-ci n'est pas censé affecter le droit de propriété du sous-sol. Le pouvoir de l'État sur l'octroi de la concession minière s'en retrouve réduit, ce qui place l'explorateur en position de force dans l'accès au sous-sol.

\section{Conclusion}

Le partage du sous-sol à des fins de stockage est régi principalement selon les mécanismes du droit minier qui mobilisent des pouvoirs régaliens importants afin d'exploiter les ressources souterraines considérées comme étant d'intérêt public. Dans un premier temps, les formations géologiques de stockage sont appropriées par l'État au nom de l'intérêt de la collectivité nationale, ce qui entraîne l'éviction du propriétaire privé de la surface et exclut de ce fait le recours à l'expropriation. Dans un second temps, les ressources souterraines sont mises à disposition des exploitants miniers, selon des règles qui les placent en position de force. Paradoxalement, les pouvoirs de l'État ont tendance à reculer, et c'est l'opérateur minier qui devient l'acteur fort dans l'accès au sous-sol, alors qu'il n'a pourtant aucun droit de propriété sur celui-ci. La notion de «richesse nationale» est donc une clé dans l'accès au sous-sol, car elle permet de mettre en valeur cet espace sur le plan industriel. Mais comment peut-on redéfinir aujourd'hui cette notion? Peut-on continuer à envisager le sous-sol comme une richesse purement industrielle ou peut-on l'appréhender au contraire comme relevant d'intérêts publics plus larges? Au vu des enjeux environnementaux du stockage géologique de déchets, qui conditionne les modes de production actuels en surface, et dont les risques sont déplacés dans l'espace et le temps, il semblerait que l'appropriation publique du sous-sol ne puisse pas aller sans une certaine dimension collective dans son utilisation. Les conditions juridiques d'implantation des exploitants en sous-sol font alors courir le risque d'une confiscation industrielle de l'espace souterrain et d'un déséquilibre au détriment d'autres intérêts. D'où la question centrale de la mise en politique du sous-sol, celle de l'intégration de cet espace dans les cadres décisionnels démocratiques. C'est une question qui engage la responsabilité des générations actuelles vis-à-vis d'elles-mêmes et surtout du futur.

\section{Références}

Billet P., 1994. La protection juridique du sous-sol en droit français, Thèse de doctorat, Lyon, université Lyon 3.

Campbell T., James R., Hutchings J., 2008. Carbon capture and storage project development: an overview of property rights acquisition, permitting, and operational liability issues, Texas Environmental Law Journal, 38, 3, 169-186.

Cuvillier T., 1902. Législation minière et contrôle des mines, Paris, Dunod.

Devaux-Charbonnel J., 1987. Droit minier des hydrocarbures : principes et applications, Paris, Éditions Technip.

Dross W., 2010. Acquisition de la propriété par union ou incorporation - Propriété du dessus et du dessous du sol, Jurisclasseur Civil Code, Fasc. Propriété, LexisNexis.

Gauché S., 2003. La formation du droit minier français, l'exemple du Dauphiné (XIV ${ }^{\mathrm{e}}$-début $\mathrm{XIX}^{\mathrm{e}}$ siècle), Revue historique de droit français et étranger, 81, 1, 75-89, https:// www.jstor.org/stable/43851382.

Gautier M.-A., 2013. L'attribution des titres miniers à l'épreuve de la réforme du Code minier, Droit de l'environnement, 202, 172.

Gillig D., 2009. Commentaire sous: Cour administrative d'appel de Nancy, 04/12/2008, n $^{\circ} 06 \mathrm{NC} 01641$, M. X et Assoc. pour la sauvegarde du sol et du sous-sol, Environnement, 3, commentaire $\mathrm{n}^{\circ} 35$.

Grave B., 2010, Carbon Capture and Storage in South Dakota : The Need for a Clear Designation of Pore Space Ownership, South Dakota Law Review, 55, 88.

Ineris, 2017. Captage et stockage géologique $d u \mathrm{CO}_{2}$ : retour d'expérience et perspectives, Rapport DRS17-164859-08281A.

Interstate Oil and Gas Compact Commission (IOGCC), 2007. $\mathrm{CO}_{2}$ Storage: A Legal and Regulatory Guide for States and 
Provinces, Task Force on Carbon Capture and Geological Storage.

Laubadère (de) A., 2000. Traité de droit administratif Administration de l'économie, Tome IV, LGDJ, Paris.

Levy J.-P., Castaldo A., 2002. Histoire du droit civil, Paris, Dalloz, Précis, $2^{\mathrm{e}}$ éd.

Personnaz J., Verrier F., Jallade P., 2004a. Droit des mines : régime des recherches minières, Jurisclasseur, LexisNexis Editions, fasc. $n^{\circ} 370$.

Personnaz J., Verrier F., Jallade P., 2004b. Droit des mines : institution des titres miniers, Jurisclasseur, LexisNexisEditions, fasc. ${ }^{\circ} 371$.

Pommier R., 1939. Le contentieux minier, Thèse de doctorat, Paris, Université de Paris, Faculté de droit.
Schellenberger T., 2014. Le droit public des utilisations du sous-sol : réflexions sur le régime juridique des stockages géologiques de déchets, Thèse de doctorat, Aix-Marseille Université.

Sillard P., 1955. Le permis exclusif de recherche d'hydrocarbure liquides et gazeux, L'Actualité Juridique du Droit Administratif, 10.

Terré F., Simler P., 2006. Droit civil - Les biens, Paris, Dalloz, Précis, $7^{\mathrm{e}}$ éd.

Vidal R., 1949. Contentieux des mines et répartition des compétences juridictionnelles, Revue du droit public et de la science politique en France et à l'étranger, 519.

Citation de l'article: Schellenberger T. Stockage géologique de déchets et droit du sous-sol : une fracture entre intérêt public et usage industriel. Nat. Sci. Soc. 29, S25-S32. 